GLASNIK MATEMATIČKI

Vol. 39(59)(2004), $235-243$

\title{
2-GROUPS WITH A SELF-CENTRALIZING ABELIAN SUBGROUP OF TYPE $(4,2)$
}

\author{
ZVONIMIR JANKO \\ University of Heidelberg, Germany
}

\begin{abstract}
In this paper we classify finite 2-groups $G$ which possess a self-centralizing abelian subgroup $A$ of type $(4,2)$. In the difficult case, where $A$ is contained in the Frattini subgroup $\Phi(G)$, we describe the structure of the group $G$ in great detail. In the other case, where $A \leq \Phi(G)$, there is an open problem.
\end{abstract}

\section{INTRODUCTION AND KNOWN RESULTS}

An abelian subgroup $A$ of a group $G$ is called "self-centralizing" in $\mathrm{G}$ if $C_{G}(A)=A$. Let $A$ be a self-centralizing abelian subgroup of order $p^{2}$ in a finite $p$-group $G$. Then, by a well known result of M. Suzuki, $G$ must be of maximal class. If, in addition, $p=2$, then $G$ is dihedral, semi-dihedral or a generalized quaternion group. However, if $p>2$, we are still very far from the determination of such $p$-groups $G$ (of maximal class). This shows that small self-centralizing abelian subgroups have stronger influence on the structure of 2 -groups $\mathrm{G}$ than in case of $p$-groups $\mathrm{G}$ for $p>2$.

It is natural to try to classify finite 2 -groups $G$ which possess a selfcentralizing abelian subgroup $A$ of order 8 . If $A \cong E_{8}$ is elementary abelian of order 8, then such groups $G$ are classified in Janko [2]. The next important case, where $A \cong C_{4} \times C_{2}$ is abelian of type $(4,2)$ will be handled in this paper. The possibility $A \leq \Phi(G)$ (i.e., $A$ is contained in the Frattini subgroup $\Phi(G)$ of $G$ ) is considered in Theorems 2.1 and 2.2 which describe the structure of such groups $G$ in great detail. The case $A \not \leq \Phi(G)$ is studied in Theorems 2.3 and 2.4. The 2-groups $G$ appearing in Theorem 2.3 have been classified in

2000 Mathematics Subject Classification. 20D15.

Key words and phrases. Finite 2-groups, self-centralizing subgroups, Frattini subgroups, minimal nonabelian 2-groups, dihedral, semi-dihedral, generalized quaternian 2groups. 
Janko [2], [3], and [4]. However, the classification of 2-groups $G$ which appear in Theorem 2.4 is still an open question.

All groups considered here are finite and our notation is standard. For convenience, we state here some known results which are used in this paper.

Proposition 1.1. (Janko [2, Proposition 1.10]) Let $\tau$ be an involutory automorphism acting on an abelian 2-group $B$ so that $C_{B}(\tau)=W_{0}$ is contained in $\Omega_{1}(B)$. Then $\tau$ acts invertingly on $\mho_{1}(B)$ and on $B / W_{0}$.

Proposition 1.2. (Janko [1, Theorem 1.1]) Let $G$ be a finite 2-group which does not have a normal elementary abelian subgroup of order 8. Suppose that $G$ is neither abelian nor of maximal class. Then $G$ possesses a normal metacyclic subgroup $N$ such that $C_{G}\left(\Omega_{2}(N)\right) \leq N$ and one of the following holds:

(a) $|G / N| \leq 4$ and either $\Omega_{2}(N)$ is abelian of type $(4,4)$ or $N$ is abelian of type $\left(2^{j}, 2\right), j \geq 2$.

(b) $G / N \cong D_{8}$ and

(b1) $N$ is either abelian of type $\left(2^{k}, 2^{k+1}\right), k \geq 1$ or of type $\left(2^{l}, 2^{l}\right), l \geq 2$ or

(b2) $N$ is minimal nonabelian, $\Omega_{2}(N)$ is abelian of type $(4,4)$ and more precisely $N=\left\langle a, b \mid a^{2^{m}}=b^{2^{n}}=1, a^{b}=a^{1+2^{m-1}}\right\rangle$, where $m=n$ with $n \geq 3$ or $m=n+1$ with $n \geq 2$.

Proposition 1.3. (Janko [1, Proposition 1.10])

Let $G$ be a 2-group with a metacyclic normal subgroup $N$. Suppose that $N$ has a $G$-invariant four-subgroup $N_{0}$ which is not contained in $Z(G)$ but there is no $G$-invariant cyclic subgroup of order 4 contained in $N$. If $N$ is abelian, then $N$ is of type $\left(2^{n}, 2^{n}\right)$ or $\left(2^{n+1}, 2^{n}\right)$, where $n \geq 1$. If $N$ is nonabelian, then $N$ is minimal nonabelian and moreover $N=\left\langle a, b \mid a^{2^{m}}=b^{2^{n}}=1, a^{b}=a^{1+2^{m-1}}\right\rangle$, where $n \geq 2$ and $m=n$ or $m=n+1$.

\section{NeW RESUlts}

We classify finite 2 -groups $G$ which possess an abelian subgroup $A \cong$ $C_{4} \times C_{2}$ such that $C_{G}(A)=A$. If $A$ is normal in $G$, then $G / A$ is isomorphic to a subgroup of $D_{8}$. Therefore we may assume in the sequel that such a subgroup $A$ is not normal in $G$. We investigate first the case where $A \leq \Phi(G)$ and prove the following basic result.

TheOREM 2.1. Let $G$ be a 2-group which possesses a self-centralizing abelian subgroup $A$ of type $(4,2)$ but $G$ does not possess any self-centralizing abelian normal subgroup of type $(4,2)$. If $A \leq \Phi(G)$, then $G$ has no normal elementary abelian subgroup of order 8 .

Proof. We assume $A \leq \Phi(G)$. Let $W_{0}=\Omega_{1}(A)$ so that $W_{0} \cong E_{4}$ and $Z(\Phi(G))<A$. If $Z(\Phi(G)) \nsupseteq W_{0}$, then $Z(\Phi(G))$ is cyclic and so $\Phi(G)$ is also cyclic (Burnside), a contradiction. 
We have proved that $Z(\Phi(G))=W_{0}$ (since $A$ is not normal in $G$ ) and so $W_{0}$ is a normal four-subgroup in $G$. Obviously, $W_{0}$ is the unique normal four-subgroup of $G$. Indeed, if $W_{1}$ were another normal four-subgroup of $G$, then $A$ does not centralize $W_{1}$ and so $C_{G}\left(W_{1}\right)$ is a maximal subgroup of $G$ not containing $A$, contrary to our assumption that $A \leq \Phi(G)$. Since $A<\Phi(G)$ and $|G / \Phi(G)| \geq 4$, so $|G| \geq 2^{6}$.

Assume that $W_{0} \leq Z(G)$. Let $B$ be a normal subgroup of $G$ such that $W_{0}<B \leq \Phi(G)$ and $\left|B: W_{0}\right|=2$. Then $G$ stabilizes the chain $B>W_{0}>\{1\}$ and so $G / C_{G}(B)$ is elementary abelian. In particular, $\Phi(G) \leq C_{G}(B)$ which contradicts the fact that $Z(\Phi(G))=W_{0}$. Hence $\left|G: C_{G}\left(W_{0}\right)\right|=2$ and so $Z(G)=\langle z\rangle<W_{0}$. Set $W_{0}=\langle z, u\rangle$ and $T=C_{G}\left(W_{0}\right)$. For each $x \in G-T$, $u^{x}=u z$ and for each $a \in A-W_{0}, C_{T}(a)=A$.

Suppose that $A_{1}$ is a normal elementary abelian subgroup of order 16 of $T=C_{G}\left(W_{0}\right)$. Take an element $a \in A-W_{0}$. Since $a^{2} \in W_{0} \leq Z(T)$, the element $a$ induces on $A_{1}$ an automorphism of order $\leq 2$ and so $\left|C_{A_{1}}(a)\right| \geq 4$. Since $C_{T}(a)=A$, we get $C_{A_{1}}(a)=W_{0}$ and $A \cap A_{1}=W_{0}$. Set $C=A A_{1}$ so that $|C|=2^{5}$ and $\left|C: A_{1}\right|=2$.

Set $\langle s\rangle=\mho_{1}(A)<W_{0}$ and we see that all four elements in $A-W_{0}$ are square roots of $s$ in $C$. Let $a a_{1}\left(a \in A-W_{0}, a_{1} \in A_{1}\right)$ be any square root of $s$ in $C$. Then we compute

$$
s=\left(a a_{1}\right)^{2}=a a_{1} a a_{1}=a^{2}\left(a^{-1} a_{1} a\right) a_{1}=s a_{1}^{a} a_{1},
$$

and so $a_{1}^{a}=a_{1}$ which implies that $a_{1} \in W_{0}$. It follows that four elements in $A-W_{0}$ are the only square roots of $s$ in $C$ and they form a single conjugate class in $C$ (since $C_{T}(a)=A$ ). In particular, $A$ is normal in $N_{T}(C)$ (since $T$ centralizes $s$ ) which implies $C=T$ and $|G|=2^{6}$.

Let $b$ be any element in $C-A_{1}=T-A_{1}$ so that $b=a_{1} a$ for an $a \in A-W_{0}$ and $a_{1} \in A_{1}$. Hence $C_{A_{1}}(b)=C_{A_{1}}(a)=W_{0}$. In particular, $b^{2} \in W_{0}$ and so four elements in $\left\langle W_{0}, b\right\rangle-W_{0}$ are either involutions (in case $b^{2}=1$ ) or square roots of the involution $b^{2}$. Conversely, let $b x\left(x \in A_{1}\right)$ be any square root of $b^{2}$ (or an involution in $C-A_{1}$ when $b^{2}=1$ ). Then we compute

$$
b^{2}=(b x)^{2}=b x b x=b^{2}\left(b^{-1} x b\right) x=b^{2} x^{b} x,
$$

which implies $x^{b}=x$ and so $x \in W_{0}$. Hence four elements in $\left\langle W_{0}, b\right\rangle-W_{0}$ are all possible square roots of $b^{2}$ (or involutions) in $C-A_{1}$. There are exactly 16 elements in $C-A_{1}$ and so each involution in $W_{0}$ has exactly four square roots in $C-A_{1}$ and in addition there are exactly four involutions in $C-A_{1}$. Also note that $Z(C)=Z(T)=W_{0}$ and so $A_{1}$ is a characteristic subgroup of $C=T$ and therefore $A_{1}$ is also normal in $G$.

Let $z \in W_{0}$ be the central involution in $G$. By the previous paragraph, there are exactly four square roots of $z$ in $C$ and if $c$ is one of them, then $\left\langle W_{0}, c\right\rangle \cong C_{4} \times C_{2}$ and all square roots of $z$ in $C=T$ lie in $\left\langle W_{0}, c\right\rangle$. Hence $\left\langle W_{0}, c\right\rangle-W_{0}$ is a normal subset in $G$ and so $\left\langle W_{0}, c\right\rangle$ is normal in $G$. But 
$C_{C}(c)=\left\langle W_{0}, c\right\rangle$ and $C_{G}\left(W_{0}\right)=C=T$. Thus $\left\langle W_{0}, c\right\rangle$ is a self-centralizing (in $G$ ) normal abelian subgroup of $G$ of type $(4,2)$, a contradiction.

We have proved that $T=C_{G}\left(W_{0}\right)$ does not possess a normal elementary abelian subgroup of order 16. Suppose that $G$ has a normal elementary abelian subgroup $A_{2}$ of order 16 . Then $A_{2} \not \leq T$ and so $A_{2} \cap T$ is normal in $G$ and $A_{2} \cap T \cong E_{8}$. Let $W^{*}$ be a $G$-invariant four-subgroup contained in $A_{2} \cap T$. Because of the uniqueness of $W_{0}$, we have $W^{*}=W_{0}$. But then $C_{G}\left(W_{0}\right) \geq\left\langle T, A_{2}\right\rangle=G$, a contradiction. We have proved that $G$ does not have a normal elementary abelian subgroup of order 16 .

Suppose that $G$ has a normal elementary abelian subgroup $E \cong E_{8}$. If $E \not \leq T$, then $E \cap T$ is a normal four-subgroup of $G$ and so $E \cap T=W_{0}$ (because of the uniqueness of $\left.W_{0}\right)$. But then $C_{G}\left(W_{0}\right) \geq\langle T, E\rangle=G$, a contradiction.

We have proved that $E \leq T$ and $E>W_{0}$ (because of the uniqueness of $\left.W_{0}\right)$ and so $A \cap E=W_{0}$. Let $B$ be a maximal $G$-invariant abelian subgroup containing $E$. Since $B \geq E>W_{0}$, we have $B \leq T$ (otherwise, $C_{G}\left(W_{0}\right) \geq$ $\langle T, B\rangle=G$, a contradiction). We know that $C_{G}(B)=B$.

Suppose that $B=E$. Then $E$ is a normal elementary abelian selfcentralizing subgroup of $G$ and so $G / E \cong D_{8}$ (since $|G| \geq 2^{6}$ ). Set $V_{0}=A E$ so that $V_{0} / E=\Phi(G / E)$ and therefore $V_{0}$ is normal in $G$. Indeed, if $M / E$ is a maximal subgroup of $G / E$, then $A \leq M$ since $A \leq \Phi(G)$. Since $T$ stabilizes the chain $E>W_{0}>\{1\}$, it follows that $T / E \cong E_{4}$. Take an element $a \in A-W_{0}$ so that four elements in $A-W_{0}$ are square roots of $a^{2} \neq 1$. Let $a e^{\prime}\left(e^{\prime} \in E\right)$ be any square root of $a^{2}$ in $V_{0}$. Then we have

$$
a^{2}=\left(a e^{\prime}\right)^{2}=a e^{\prime} a e^{\prime}=a^{2}\left(e^{\prime}\right)^{a} e^{\prime},
$$

which gives $\left(e^{\prime}\right)^{a}=e^{\prime}$ and so $e^{\prime} \in W_{0}$ since $C_{T}(a)=A$. It follows that $\left\{A-W_{0}\right\}$ is the set of all square roots of $a^{2}$ in $V_{0}$ and so $A$ is normal in $T$. Since $A$ is not normal in $G$, we have for every $x \in G-T, A^{x} \neq A$. Since $A^{x} \leq V_{0}$ and $A \cap A^{x}=W_{0}$, we have

$$
V_{0}-E=E a=\left(A-W_{0}\right) \cup\left(A^{x}-W_{0}\right)
$$

and so $x$ sends four elements in $A-W_{0}$ onto four elements in $A^{x}-W_{0}$. But $G / E \cong D_{8}$ and so (since $T / E \cong E_{4}$ and $V_{0} / E=\Phi(G / E)$ ) there is $y \in G-T$ with $y^{2} \in V_{0}-E$. This is a contradiction since $y$ centralizes $y^{2} \in V_{0}-E$ and so $y$ normalizes $A$ and $A^{x}$.

We have proved that $B>E$ and so $|B| \geq 2^{4}$. Since $\Omega_{1}(B)=E, B$ is an abelian group of rank 3. Take an element $a \in A-W_{0}$. Since $a^{2} \in W_{0} \leq Z(T)$, $a$ induces an involutory automorphism on $B$ with $C_{B}(a)=W_{0} \leq \Omega_{1}(B)$ (since $C_{T}(a)=A$ ). Applying Proposition 1.1, we see that $a$ inverts $\mho_{1}(B)$ and $B / W_{0}$. Suppose that some $e \in E-W_{0}$ is a square in $B$. Then $a$ inverts (centralizes) $e$, a contradiction. Suppose that each involution in $W_{0}$ is a square in $B$. Then

$$
\Omega_{2}(B)=\left\langle b_{1}\right\rangle \times\left\langle b_{2}\right\rangle \times\left\langle b_{3}\right\rangle,
$$


where $o\left(b_{1}\right)=o\left(b_{2}\right)=4, b_{3}$ is an involution in $E-W_{0}$, and $\left\langle b_{1}^{2}, b_{2}^{2}\right\rangle=W_{0}$. By the above, $b_{1}^{a}=b_{1}^{-1} w$ with $w \in W_{0}$ and so

$$
b_{1}^{a}=b_{1}^{-1} w=b_{1}\left(b_{1}^{2} w\right)=b_{1} w_{1},
$$

where $w_{1} \in W_{0}$. Similarly, $b_{2}^{a}=b_{2} w_{2}$ and $b_{3}^{a}=b_{3} w_{3}$ with $w_{2}, w_{3} \in W_{0}$. Since $C_{\Omega_{2}(B)}(a)=W_{0}, w_{1}, w_{2}, w_{3}$ must be three distinct involutions in $W_{0}$. But then

$$
\left(b_{1} b_{2} b_{3}\right)^{a}=\left(b_{1} b_{2} b_{3}\right)\left(w_{1} w_{2} w_{3}\right)=b_{1} b_{2} b_{3} \in \Omega_{2}(B)-W_{0},
$$

a contradiction. Hence we have $B=\langle b\rangle \times\langle w\rangle \times\langle e\rangle$, where $o(b)=2^{m}, m>1$, $b^{2^{m-1}}=z,\langle z\rangle=Z(G)=\mho_{m-1}(B), W_{0}=\langle z, w\rangle$, and $e \in E-W_{0}$. Also we set $v=b^{2^{m-2}}$ so that $o(v)=4, v^{2}=z$, and $\Omega_{2}(B)=\langle E, v\rangle$.

Set $V=A B$ and assume that $V<T$. Set $\tilde{V}=N_{T}(V)$ so that $|\tilde{V}: V| \geq 2$. Obviously, $V-B=a B\left(a \in A-W_{0}\right)$ is a normal subset in $V^{*}=N_{G}(V) \geq \tilde{V}$. Set $a^{2}=w^{\prime} \in W_{0}$ so that four elements in $A-W_{0}$ are square roots of $w^{\prime}$ in $V-B$. An element $a b^{\prime} \in V-B\left(b^{\prime} \in B\right)$ is a square root of $w^{\prime}$ if and only if

$$
w^{\prime}=\left(a b^{\prime}\right)^{2}=a b^{\prime} a b^{\prime}=a^{2}\left(b^{\prime}\right)^{a} b^{\prime}=w^{\prime}\left(b^{\prime}\right)^{a} b^{\prime}
$$

or $\left(b^{\prime}\right)^{a}=\left(b^{\prime}\right)^{-1}$. If $\left(b^{\prime}\right)^{a}=\left(b^{\prime}\right)^{-1}$ and $\left(b^{\prime \prime}\right)^{a}=\left(b^{\prime \prime}\right)^{-1}\left(b^{\prime}, b^{\prime \prime} \in B\right)$, then

$$
\left(b^{\prime} b^{\prime \prime}\right)^{a}=\left(b^{\prime}\right)^{a}\left(b^{\prime \prime}\right)^{a}=\left(b^{\prime}\right)^{-1}\left(b^{\prime \prime}\right)^{-1}=\left(b^{\prime} b^{\prime \prime}\right)^{-1}
$$

and so the set $B_{0}$ of elements of $B$ which are inverted by $a$ is a subgroup of $B$. The number of square roots of $w^{\prime}$ in $V-B$ is equal $\left|B_{0}\right|$. Obviously, $B_{0} \cap E=$ $W_{0}$ since $C_{T}(a)=A$. We have $|B|=2^{m+2}(m>1)$, so $|V-B|=2^{m+2}$ and therefore $\left|B_{0}\right| \leq 2^{m+1}$. All conjugates of $a$ in $\tilde{V}$ lie in $V-B$ and all these conjugates are square roots of $w^{\prime}$ since $\tilde{V} \leq T$ and $T$ centralizes $w^{\prime} \in W_{0}$. Since $C_{\tilde{V}}(a)=A$, so $|\tilde{V}: A| \leq 2^{m+1}$. But $|V|=2^{m+3}$ and so $|\tilde{V}|=2^{m+4}$, $|\tilde{V}: V|=2$, and $\left|B_{0}\right|=2^{m+1}$. It follows that $B_{0}$ covers $B / E$ and so we may choose $b \in B_{0}$ so that $B_{0}=\langle b\rangle \times\langle w\rangle$, where $w \in W_{0}-\langle z\rangle$ (since $b^{2^{m-1}}=z$ ).

Suppose for a moment that $\tilde{V}=N_{T}(V)=N_{G}(V)$. Then looking at $G / B$, we get (by a well known result of M. Suzuki) that $G / B$ is of maximal class and $V / B$ is a non-central subgroup of order 2 in $G / B$. Let $R / B$ be a cyclic subgroup of index 2 in $G / B$. But then $R$ is a maximal subgroup of $G$ and $R \cap V=B$. In particular, $A \not \leq R$, contrary to our assumption $A \leq \Phi(G)$. We have proved that $V^{*}=N_{G}(V)>\tilde{V}=N_{T}(V)$ so that $\left|V^{*}: \tilde{V}\right|=2, G=T V^{*}$, and $T \cap V^{*}=\tilde{V}$.

Assume that $C_{V^{*}}(a)=A=C_{\tilde{V}}(a)$ and take an element $y \in V^{*}-\tilde{V}$ so that $y \in G-T$. Then all $2^{m+2}$ elements in $V-B$ form a single conjugate class in $V^{*}$. Since $w^{\prime}$ has exactly $2^{m+1}$ square roots in $V-B$, it follows that $w^{\prime}=a^{2} \neq z($ since $\langle z\rangle=Z(G))$ and so $\left(w^{\prime}\right)^{y}=w^{\prime} z$. Hence $y$ sends $2^{m+1}$ square roots of $w^{\prime}$ in $V-B$ onto $2^{m+1}$ square roots of $w^{\prime} z$ in $V-B$. Since $e \notin B_{0}\left(e \in E-W_{0}\right)$, we have

$$
(a e)^{2}=w^{\prime} z=a e a e=a^{2} e^{a} e=w^{\prime} e^{a} e,
$$


and so $e^{a}=e z$. On the other hand, $a$ inverts $B_{0}=\langle b, w\rangle$ and so setting $v=b^{2^{m-2}}$, we get $v^{2}=z$ and $v^{a}=v^{-1}=v z$. But then $(e v)^{a}=e z v z=e v$, contrary to $C_{B}(a)=W_{0}$. We have proved that $\tilde{A}=C_{V^{*}}(a)>A$, where $|\tilde{A}: A|=2$ and $\tilde{A} \cap T=A$.

Take an element $y \in \tilde{A}-A$ so that $y$ acts non-trivially on $W_{0}$. But $y$ centralizes $a$ and so $y$ centralizes $a^{2}=w^{\prime}$ which gives $w^{\prime}=z=b^{2^{m-1}}$, where $\langle z\rangle=Z(G)$. Suppose $y^{2} \in W_{0}$ so that $\left\langle W_{0}, y\right\rangle \cong D_{8}$. In that case there are involutions in $\left\langle W_{0}, y\right\rangle-W_{0}$ and so we may assume that $y$ is an involution. Act with the involution $y$ on $E$. Since $y$ acts non-trivially on $W_{0}$ and $\left|C_{E}(y)\right|=4$, there is an involution $e^{\prime} \in E-W_{0}$ with $\left(e^{\prime}\right)^{y}=e^{\prime}$. We have $1 \neq\left[a, e^{\prime}\right] \in W_{0}$ and so

$$
\left[a, e^{\prime}\right]^{y}=\left[a^{y},\left(e^{\prime}\right)^{y}\right]=\left[a, e^{\prime}\right],
$$

which gives $\left[a, e^{\prime}\right]=z$ or $\left(e^{\prime}\right)^{a}=e^{\prime} z$. But $a$ inverts $B_{0}$ and so $v^{a}=v^{-1}=v z$ (where $v=b^{2^{m-2}}$ ) which gives

$$
\left(v e^{\prime}\right)^{a}=v z e^{\prime} z=v e^{\prime} \notin W_{0} .
$$

This is a contradiction since $C_{B}(a)=W_{0}$.

We have proved that for each $y \in \tilde{A}-A, y^{2} \in A-W_{0}$ and so we may assume that $y^{2}=a$. Since $a^{2}=z$ centralizes $E$, it follows that $y$ induces on $E$ an automorphism of order 4 and so $C_{E}(y)=\langle z\rangle$. Hence for an $e \in E-W_{0}$, we get

$$
e^{y}=e w_{0} \text { with } w_{0} \in W_{0}-\langle z\rangle \text { and } w_{0}^{y}=w_{0} z
$$

This gives

$$
e^{a}=e^{y^{2}}=\left(e w_{0}\right)^{y}=\left(e w_{0}\right)\left(w_{0} z\right)=e z .
$$

On the other hand, $a$ inverts $B_{0}=\left\langle b, w_{0}\right\rangle$ and so $v^{a}=v^{-1}=v z$. This gives

$$
(v e)^{a}=v z e z=v e \notin W_{0},
$$

contrary to $C_{B}(a)=W_{0}$.

The contradiction in the previous paragraph shows that we must have $\mathrm{V}=\mathrm{AB}=\mathrm{T}$. We set again $a^{2}=w^{\prime} \in W_{0}\left(a \in A-W_{0}\right)$ and $v=b^{2^{m-2}}(m>1)$ so that $v^{2}=z$, where $\langle z\rangle=Z(G)$ and $B=\langle E, b\rangle$. We have $|G / B|=4$. If $G / B \cong E_{4}$, then $\Phi(G) \leq B$ and this contradicts our assumption $A \leq \Phi(G)$. Hence $G / B \cong C_{4}$ and $G / B$ acts faithfully on $E$ (since $T=B\langle a\rangle$ and $a$ acts non-trivially on $E$ ). If $y \in G-T$, then for an element $e \in E-W_{0}$, we have $e^{y}=e w$ with $w \in W_{0}-\langle z\rangle$ and $w^{y}=w z$. Then we compute

$$
e^{y^{2}}=(e w)^{y}=(e w)(w z)=e z .
$$

But $y^{2} \in T-B$ and $T=B\langle a\rangle$ and so $a$ acts in the same way on $E$ as the element $y^{2}$ which implies $e^{a}=e z$. By Proposition 1.1, $a$ inverts $\mho_{1}(B)$. If $m>2$, then $v \in \mho_{1}(B)$ and so $v^{a}=v^{-1}=v z$. We get in that case $(e v)^{a}=e z v z=e v$ which contradicts to $C_{B}(a)=W_{0}$. 
We have proved that we must have $m=2$ and so $b=v,|B|=2^{4}$, and $|G|=2^{6}$. The argument of the previous paragraph shows that $a$ does not invert any element (of order 4 ) in $B-E$ and so the subgroup $B_{0}$ of all elements of $B$ inverted by $a$ is equal $B_{0}=W_{0}$. Indeed, if $a$ inverts an element $s \in B-E$, then $s^{a}=s^{-1}=s z$ and so $(e s)^{a}=e z s z=e s$, contrary to $C_{B}(a)=W_{0}$. Hence $a^{2}=w^{\prime}$ has exactly $\left|B_{0}\right|=4$ square roots in $T-B=V-B=(A B)-B$ and they all lie in $A-W_{0}$. Since $\left\langle A-W_{0}\right\rangle=A, A$ is normal in $T=A B$. For each $x \in T-B, x^{2} \in W_{0}$ since $C_{B}(x)=C_{B}(a)=W_{0}$, and $x$ (acting in the same way on $B$ as the element $a$ ) inverts on $B$ exactly the elements of $W_{0}$. It follows that $x^{2}$ has exactly four square roots in $T-B$. Hence each involution in $W_{0}$ has exactly four square roots in $T-B$ and (since $\left.|T-B|=16\right) T-B$ contains exactly four involutions.

Let $a^{\prime} \in T-B$ so that $\left(a^{\prime}\right)^{2}=z$. Set $A^{*}=W_{0}\left\langle a^{\prime}\right\rangle \cong C_{4} \times C_{2}$ and $A^{*}$ is normal in $G$. Indeed, $\langle z\rangle=Z(G)$ and so $G$ normalizes the subset $\left\{\left(W_{0}\left\langle a^{\prime}\right\rangle\right)-W_{0}\right\}$ of all square roots of $z$ in $T-B$. We have $C_{G}\left(W_{0}\right)=$ $T$ and $C_{T}\left(a^{\prime}\right)=\left\langle a^{\prime}\right\rangle C_{B}\left(a^{\prime}\right)$. But $C_{B}\left(a^{\prime}\right)=C_{B}(a)=W_{0}$ and so $A^{*}$ is a self-centralizing abelian normal subgroup of type $(4,2)$ in $G$. This is a final contradiction and our theorem is proved.

In our next result we shall determine the structure of the groups appearing in Theorem 2.1.

TheOREM 2.2. Let $G$ be a 2-group which possesses a self-centralizing abelian subgroup $A$ of type (4,2) but $G$ does not possess any self-centralizing abelian normal subgroup of type (4,2). If $A \leq \Phi(G)$, then $G$ has the following properties:

(i) $G$ has no normal elementary abelian subgroup of order 8.

(ii) $G$ has the unique normal four-subgroup $W_{0}=\Omega_{1}(A)$.

(iii) $G$ has a normal metacyclic subgroup $N$ such that $\Omega_{2}(N)=W$ is abelian of type $(4,4), C_{G}(W) \leq N, \Omega_{1}(W)=W_{0}, C_{G}\left(W_{0}\right) \geq N$, $\mid G: C_{G}\left(W_{0} \mid=2\right.$, and $G / N \cong C_{4}$ or $D_{8}$.

(iv) $N$ is either abelian of type $\left(2^{k}, 2^{k+1}\right)$ or $\left(2^{k}, 2^{k}\right), k \geq 2$, or $N$ is minimal nonabelian and more precisely

$$
N=\left\langle a, b \mid a^{2^{m}}=b^{2^{n}}=1, a^{b}=a^{1+2^{m-1}}\right\rangle,
$$

where either $m=n$ with $n \geq 3$ or $m=n+1$ with $n \geq 2$.

Proof. Suppose that our 2-group $G$ satisfies all the assumptions of our theorem together with $A \leq \Phi(G)$. By Theorem 2.1, $G$ has no normal elementary abelian subgroup of order 8 . By the first three paragraphs of the proof of Theorem 2.1, we know that $W_{0}=Z(\Phi(G))=\Omega_{1}(A)$ is the unique normal four-subgroup of $G,\left|G: C_{G}\left(W_{0}\right)\right|=2, Z(G)$ is of order 2 , and $|G| \geq 2^{6}$. Set $W_{0}=\langle z, u\rangle, T=C_{G}\left(W_{0}\right)$, where $\langle z\rangle=Z(G)$. For each $t \in A-W_{0}$, $C_{T}(t)=A$. 
We apply now Proposition 1.2, since $G$ is neither abelian nor of maximal class. It follows that $G$ possesses a normal metacyclic subgroup $N$ such that $C_{G}\left(\Omega_{2}(N)\right) \leq N, G / N$ is isomorphic to a subgroup of $D_{8}$ and $W=\Omega_{2}(N)$ is abelian of type $(4,2)$ or $(4,4)$. In any case, $W_{0}=\Omega_{1}(W)=\Omega_{1}(N)$ is the unique normal four-subgroup of $G$ and $W_{0} \leq \leq Z(G)$. If $A \leq N$, then $A \leq W=\Omega_{2}(N)$. Since $C_{G}(A)=A$, we have $A=W$ and then $A$ is normal in $G$, a contradiction. Hence $A \not \leq N$ and so $A \cap N=W_{0}$. Since $A \leq \Phi(G)$, $G / N$ is not elementary abelian. Hence $G / N$ is isomorphic to $C_{4}$ or $D_{8}$. If $T=C_{G}\left(W_{0}\right)$ does not contain $N$, then $T$ covers $G / N$ and $G / N$ acts faithfully on $W$ (since $\left.C_{G}(W) \leq N\right)$. In that case $C_{G}\left(W_{0}\right) / C_{N}\left(W_{0}\right)$ cannot contain elements of order 4 (since that group centralizes $W_{0}=\Omega_{1}(W)$ ) and so $G / N$ is elementary abelian, a contradiction. Hence $T=C_{G}\left(W_{0}\right) \geq N$.

Assume that $G / N \cong C_{4}$. If $N$ is abelian of type $\left(2^{j}, 2\right), j \geq 2$, then $G / N$ acts faithfully on $\Omega_{2}(N) \cong C_{4} \times C_{2}$. If $N>\Omega_{2}(N)$, then there is a characteristic cyclic subgroup $Z \cong C_{4}$ of $N$ so that $Z$ is normal in $G$. But then $\left|G: C_{G}(Z)\right| \leq 2$ and therefore $A$ (being contained in $\Phi(G)$ ) centralizes $Z$, a contradiction. Thus $N=\Omega_{2}(N)$ is a normal abelian self-centralizing subgroup of type $(4,2)$ in $G$, a contradiction. We have proved that $\Omega_{2}(N)=W$ is abelian of type $(4,4)$. Suppose that $Z^{*}$ is a $G$-invariant cyclic subgroup of order 4 contained in $N$. But then again $A$ centralizes $Z^{*}$, a contradiction. Hence there is no such $Z^{*}$ and so we may apply Proposition 1.3. It follows that $N$ is either abelian of type $\left(2^{n}, 2^{n}\right)$ or $\left(2^{n+1}, 2^{n}\right)$ with $n \geq 2$ or $N$ is minimal non-abelian and more precisely

$$
N=\left\langle a, b \mid a^{2^{m}}=b^{2^{n}}=1, a^{b}=a^{1+2^{m-1}}\right\rangle,
$$

where either $m=n$ with $n \geq 3$ or $m=n+1$ with $n \geq 2$.

Assume that $G / N \cong D_{8}$. The structure of $N$ in that case is already determined by Proposition 1.2. The minimal case $N \cong C_{4} \times C_{2}$ cannot occur because in that case $N$ would be a self-centralizing normal abelian subgroup of type $(4,2)$ of $G$, a contradiction.

Finally, we consider the case where $A \not \leq \Phi(G)$.

THEOREM 2.3. Let $G$ be a 2-group which possesses a self-centralizing abelian subgroup $A$ of type $(4,2)$. If $\Omega_{1}(A) \not \leq \Phi(G)$, then $G$ possesses an involution $t$ such that $C_{G}(t)=\langle t\rangle \times D$, where $D$ is isomorphic to one of the following groups:

$$
C_{4}, D_{8}, Q_{2^{n}}, \quad n \geq 3, \quad \text { or } \quad S D_{2^{m}}, \quad m \geq 4 .
$$

Such groups $G$ have been classified in Janko [2], [3], and [4].

Proof. Suppose that $\Omega_{1}(A) \not \leq \Phi(G)$. There is a maximal subgroup $M$ of $G$ such that $A-M$ contains an involution $t$. It follows that $A_{0}=A \cap M \cong C_{4}$ and $C_{G}(t)=\langle t\rangle \times D$, where $D=C_{M}(t) \geq A_{0}$. We have $C_{D}\left(A_{0}\right)=A_{0}$ and so 
(by a well known result of M. Suzuki) either $D=A_{0} \cong C_{4}$ or $D$ is a 2-group of maximal class. In the second case $D$ is isomorphic to

$$
D_{8}, \quad Q_{2^{n}}, \quad n \geq 3, \quad \text { or } \quad S D_{2^{m}}, \quad m \geq 4 .
$$

THEOREM 2.4. Let $G$ be a 2-group which possesses a self-centralizing abelian subgroup $A$ of type (4,2). If $W_{0}=\Omega_{1}(A) \leq \Phi(G)$ but $A \not \leq \Phi(G)$, then for any $a \in A-W_{0}, C_{G}(a)=\langle a\rangle * M_{0},\left|\langle a\rangle \cap M_{0}\right|=2$, where $M_{0}=W_{0}$ or $M_{0}$ is isomorphic to one of the following groups:

$$
D_{2^{n}}, \quad n \geq 3, \quad \text { or } S D_{2^{m}}, \quad m \geq 4 \text { and } G>C_{G}(a) .
$$

Proof. Let $M$ be a maximal subgroup of $G$ which does not contain $A$. Then $A \cap M=W_{0}=\Omega_{1}(A)$. Let $a \in A-W_{0}$ so that $G=M\langle a\rangle$ and $C_{G}(a)=\langle a\rangle C_{M}(a)$ with $C_{M_{0}}\left(W_{0}\right)=W_{0}$, where $M_{0}=C_{M}(a)$. By a result of M. Suzuki, either $M_{0}=W_{0}$ or $M_{0}$ is of maximal class. In the second case, $M_{0}$ is isomorphic to one of the following groups:

$$
D_{2^{n}}, \quad n \geq 3, \quad \text { or } \quad S D_{2^{m}}, \quad m \geq 4 .
$$

If $G=C_{G}(a)$, then $\Phi(G)=\Phi\left(C_{G}(a)\right)=\Phi\left(M_{0}\right)$ is cyclic, contrary to our assumption.

REMARK 2.5. A classification of the groups of Theorem 2.4 is an open question.

\section{REFERENCES}

[1] Z. Janko, Finite 2-groups with no normal elementary abelian subgroup of order 8 , J.Algebra 246 (2001), 951-961.

[2] Z. Janko, Finite 2-groups with a self-centralizing elementary abelian subgroup of order 8, J.Algebra 269 (2003), 189-214.

[3] Z. Janko, Finite 2-groups with small centralizer of an involution, J.Algebra 241 (2001), $818-826$.

[4] Z. Janko, Finite 2-groups with small centralizer of an involution, 2, J.Algebra 245 (2001), 413-429.

Z. Janko

Mathematical Institute

University of Heidelberg

69120 Heidelberg

Germany

E-mail: janko@mathi.uni-heidelberg.de

Received: 23.09.2003. 